\title{
Single nucleotide polymorphism mining and nucleotide sequence analysis of Mx1 gene in exonic regions of Japanese quail
}

\author{
Diwesh Kumar Niraj ${ }^{1}$, Pushpendra Kumar ${ }^{1}$, Chinmoy Mishra ${ }^{2}$, Raj Narayan ${ }^{3}$, Tarun Kumar Bhattacharya ${ }^{4}$, \\ Kush Shrivastava ${ }^{1}$, Bharat Bhushan ${ }^{1}$, Ashok Kumar Tiwari ${ }^{5}$, Vishesh Saxena ${ }^{4}$, Nihar Ranjan Sahoo ${ }^{1}$ and Deepak Sharma ${ }^{1}$ \\ 1. Animal Genetics Division, Indian Veterinary Research Institute, Izatnagar, Bareilly - 243 122, Uttar Pradesh, India; \\ 2. Department of Animal Genetics and Breeding, College of Veterinary Science and Animal Husbandry, Orissa University \\ of Agriculture and Technology, Bhubaneswar, Odisha, India; 3. Department of Avian Genetics and Breeding, Central Avian \\ Research Institute, Izatnagar, Bareilly, 243 122, Uttar Pradesh, India, 4. Directorate of Poultry Research, Rajendranagar, \\ Hyderabad, Telangana, India; 5. Standardization Division, Indian Veterinary Research Institute, Izatnagar, \\ Bareilly - 243 122, Uttar Pradesh, India \\ Corresponding author: Pushpendra Kumar, e-mail: pushpendra64@gmail.com, \\ DK: diwesh12@gmail.com, CM: drchinmoymishra@gmail.com, RN: rncari.quails@gmail.com, \\ TKB: bhattacharyatk@gmail.com, KS: kush_vet@yahoo.co.in, BB: bhushan.drbharat@gmail.com, \\ AKT: aktiwari63@yahoo.com, VS: visheshmeeta@gmail.com, NRS: vet.nihar@gmail.com, DS: ds7758@yahoo.co.in \\ Received: 02-07-2015, Revised: 31-10-2015, Accepted: 08-11-2015, Published online: 29-12-2015
}

doi: 10.14202/vetworld.2015.1435-1443 How to cite this article: Niraj DK, Kumar P, Mishra C, Narayan R, Bhattacharya TK, Shrivastava K, Bhushan B, Tiwari AK, Saxena V, Sahoo NR, Sharma D (2015) Single nucleotide polymorphism mining and nucleotide sequence analysis of $M x 1$ gene in exonic regions of Japanese quail, Veterinary World 8(12): $1435-1443$.

\begin{abstract}
Aim: An attempt has been made to study the Myxovirus resistant (Mxl) gene polymorphism in Japanese quail.

Materials and Methods: In the present, investigation four fragments viz. Fragment I of 185 bp (Exon 3 region), Fragment II of $148 \mathrm{bp}$ (Exon 5 region), Fragment III of $161 \mathrm{bp}$ (Exon 7 region), and Fragment IV of $176 \mathrm{bp}$ (Exon 13 region) of Mxl gene were amplified and screened for polymorphism by polymerase chain reaction-single-strand conformation polymorphism technique in 170 Japanese quail birds.
\end{abstract}

Results: Out of the four fragments, one fragment (Fragment II) was found to be polymorphic. Remaining three fragments (Fragment I, III, and IV) were found to be monomorphic which was confirmed by custom sequencing. Overall nucleotide sequence analysis of $M x 1$ gene of Japanese quail showed 100\% homology with common quail and more than $80 \%$ homology with reported sequence of chicken breeds.

Conclusion: The $M x l$ gene is mostly conserved in Japanese quail. There is an urgent need of comprehensive analysis of other regions of $M x l$ gene along with its possible association with the traits of economic importance in Japanese quail.

Keywords: Japanese quail, $M x 1$ gene, nucleotide sequencing, polymorphism, polymerase chain reaction-single-strand conformation polymorphism.

\section{Introduction}

The brisk increase in human population during the last few decades led to hassled research on improving production performance of livestock and poultry to meet the requirement of quality food [1]. However, increase in production performance is mostly coupled with compromised health-related traits due to their negative genetic correlation $[2,3]$. The poultry industry has been facing intimidating losses due to rise in the incidence of diseases associated with the intensive management system. Conventional vaccinations coupled with the modern managemental practices strive to protect the birds from many pathogens due to change in pathogenicity of causative agents, emerging of resistant strains, and sometime ineffective medical treatments. Hence, the current research is mostly focused on a holistic approach of a simultaneous increase in production performance along with the disease resistance traits [4].

The increasing demand for eggs and poultry meat to meet the recommended nutritional requirement

Copyright: The authors. This article is an open access article licensed under the terms of the Creative Commons Attributin License (http:// creative commons.org/licenses/by/2.0) which permits unrestricted use, distribution and reproduction in any medium, provided the work is properly cited. paves the way for rearing of alternate poultry species viz. ducks and quail which are known for their ability to produce more eggs and better meat as compared to chicken. The quail is an efficient egg and meat producer (unique flavor) having rapid growth, early sexual maturity, shorter generation interval, a higher rate of laying, early marketing age and low maintenance cost in comparison to chicken. The present concept of sustainable production requires optimum production performance along with giving appropriate weight age to disease resistance and health-related traits.

Mxl gene is an interferon-induced gene that inhibits the proliferation of avian influenza virus. However, very few reports are available on Japanese quail Mxl gene. Therefore, in the present study, we have tried to explore the genetic polymorphism of Mxl gene of Japanese quail using polymerase chain reaction-single-strand conformation polymorphism (PCR-SSCP) and nucleotide sequencing techniques.

\section{Materials and Methods}

Ethical approval

All the procedures have been conducted in accordance with the guidelines laid down by the 
Institutional Animal Ethical Committee of Indian Veterinary Research Institute.

\section{Resource population and sample collection}

Total 170 adult Japanese quail birds maintained at Central Avian Research Institute (CARI), Izatnagar, and Bareilly were selected for sample collection. About $2 \mathrm{ml}$ of blood sample was collected from each bird with EDTA as anticoagulant. The blood samples were kept in the deep freezer till DNA isolation.

\section{Amplification of exonic regions}

The genomic DNA was isolated from the collected blood samples by conventional method [5]. The quality and purity of DNA was assessed by agarose gel electrophoresis and spectrophotometer, respectively. The genomic DNA was diluted to a concentration of $50 \mathrm{ng} / \mu \mathrm{l}$. For PCR, the primers were designed $[6,7]$ on the basis of available sequences of chicken (Acc No - DQ788613) and common quail (Acc No - EF575605) in public domain of NCBI for four different regions of $M x l$ gene. The PCR reactions were carried out in a total volume of $25 \mu$ l solution containing $1 \mu \mathrm{l}$ of each forward and reverse primer (10 pmole/ $\mu \mathrm{l}$ ), $12.5 \mu 1$ mastermix (MBI Fermentas), 1-2 $\mu 1$ genomic DNA (final concentration 60-90 ng/ $\mu$ ) and nuclease free water to make final volume. The annealing temperature for different fragments was optimized (Table-1). The amplification products were separated on $1.5 \%$ agarose gel electrophoresis, stained with $5 \mu \mathrm{g} / \mathrm{ml}$ of ethidium bromide with a $100 \mathrm{bp}$ DNA ladder as molecular weight marker.

\section{Nucleotide polymorphism and DNA sequencing}

The single nucleotide polymorphisms (SNPs) of Mxl gene were identified by PCR-SSCP technique $[8,9]$. The PCR products were resolved on $15 \%$ polyacrylamide gel. About $6 \mu 1$ of PCR product and $12 \mu \mathrm{l}$ of denaturing formamide dye (formamide, $95 \%$; xylene cyanol, $0.025 \%$; bromophenol blue, $0.025 \%$; $0.5 \mathrm{M}$ EDTA, 4\%) were taken in a $0.2 \mathrm{ml}$ PCR tube and mixed properly. The mixture of $\mathrm{PCR}$ product and formamide dye were denatured at $95^{\circ} \mathrm{C}$ for $10 \mathrm{~min}$ (by keeping in hot water bath) and snap chilled on ice for $15 \mathrm{~min}$. The product was loaded in gel carefully. The electrophoresis was performed at $4^{\circ} \mathrm{C}$ for $13-16 \mathrm{~h}$ at 130 constant volts. For visualization of bands, silver staining was carried out. The pattern of DNA bands were documented by gel documentation system. The genotypes were identified, and the different SNPs were scored on banding pattern of SSCP. The gene and genotype frequencies were estimated [10]. The identified genotypes were custom sequenced and analyzed by BLAST (www.ncbi.nlm.nih.gov/BLAST). The nucleotide sequences and chromatograms were aligned and evaluated using BioEdit v7.0.5 [11]. The phylogenetic trees were constructed using MEGA 6 [12].

\section{Results and Discussion}

\section{Polymorphism of Mx1 gene}

Out of the four fragments studied, Fragment II was found to be polymorphic by SSCP. Four different SSCP genotypes viz. AA, BB, CC, and DD were identified. The genotype frequency was found to be the highest for $\mathrm{BB}$ genotype (0.44) followed by AA $(0.24), \mathrm{CC}(0.18)$, and DD (0.14) genotype. The allele frequency was found to be in the decreasing order from B, A, C, and D (Table-2). However, the SSCP analysis could not reveal any polymorphism in three fragments (Fragment I, III, and IV).

\section{Nucleotide sequence analysis}

The amplified fragments of $M x 1$ gene (Fragment I of $185 \mathrm{bp}$, Fragment II of $148 \mathrm{bp}$, Fragment III of $161 \mathrm{bp}$, and Fragment IV of $176 \mathrm{bp}$ ) of Japanese quail were custom sequenced [13] and were submitted to NCBI GenBank (KC571220, KC571221, KC571222, KC571223, KC571224, KC571225, and KC571226). All the sequences of Japanese quail as well as corresponding reported sequences of common quail, different breeds of chicken viz. RIR (NCBI Acc. No. DQ788613), SILKIE (NCBI Acc. No. DQ788614), WLH (NCBI Acc. No. DQ788615), Phasianus colchicus (Pheasant), Meleagris gallopavo (Turkey), Columba livia (Pigeon), and Lagopus lagopus (Willow ptarmigan) were aligned (Figures-1-4).

Between the Japanese quail and common quail, eight SNPs were identified in Fragment II, one SNP

Table-2: Allele-wise genotype and gene frequency in Fragment II.

\begin{tabular}{lccc}
\hline Genotype & $\begin{array}{c}\text { Genotype } \\
\text { frequency }\end{array}$ & Allele & $\begin{array}{c}\text { Gene/allele } \\
\text { frequency }\end{array}$ \\
\hline AA & 0.24 & A & 0.24 \\
BB & 0.44 & B & 0.44 \\
CC & 0.18 & C & 0.18 \\
DD & 0.14 & D & 0.14 \\
\hline
\end{tabular}

Table-1: Primer sequences and annealing temperature used to amplify $M \times 1$ gene in Japanese quail.

\begin{tabular}{|c|c|c|c|c|c|}
\hline Fragments & $\begin{array}{l}\text { Fragment } \\
\text { size }\end{array}$ & Primer & Primer sequence $\left(5^{\prime} \rightarrow 3^{\prime}\right)$ & $\begin{array}{c}\text { Primer } \\
\text { length (bp) }\end{array}$ & $\begin{array}{c}\text { Annealing } \\
\text { temperature }\left({ }^{\circ} \mathrm{C}\right)\end{array}$ \\
\hline \multirow[t]{2}{*}{ I (Exon 3) } & 185 & Forward & GCAGCAGAACACAGCTTTCA & 20 & 61 \\
\hline & & Reverse & CTAGGAAGAGCAACACCAGAC & 21 & \\
\hline \multirow[t]{2}{*}{ II (Exon 5) } & 148 & Forward & CAGGATATAGTGGCTAGCAC & 20 & 56 \\
\hline & & Reverse & GGTCATTATCTTGTGGCTGGTTCC & 24 & \\
\hline \multirow[t]{2}{*}{ III (Exon 7) } & 161 & Forward & TCCTCACTAAACCAGATCTGGTG & 23 & 59.2 \\
\hline & & Reverse & TGCTGGATTACAGAGGCCAAGGA & 24 & \\
\hline \multirow[t]{2}{*}{ IV (Exon 13) } & 176 & Forward & GCAAGCAACAGCTGCGAAAA & 20 & 61.2 \\
\hline & & Reverse & AAACCATTTCCAGGGCAAAGCTGG & 24 & \\
\hline
\end{tabular}


Coturnix japonica Coturnix coturnix White Leghorn

Silkie

Meleagris gallopav Lagopus lagopus Anas platyrhynchos Columba livia

Coturnix japonica Coturnix coturnix White Leghorn Silkie

RIR

Meleagris gallopavo Lagopus lagopus Anas platyrhynchos Columba livia

1 20 30 40 50 60 70 80 90 $\ldots \ldots|\ldots| \ldots|\ldots| \ldots|\ldots| \ldots|\ldots| \ldots|\ldots| \ldots|\ldots| \ldots|\ldots| \ldots|\ldots| \ldots|\ldots| \ldots|\ldots| \ldots|\ldots| \ldots|\ldots|$ GCAGCAGAACACAGCTTTCACAATGAATATGACATAAAGATTCGACCTTGCATTGACCTTGTTGAGAGCTTGAGAAGGCTTGATATAGGAAACAAACTGA GCAGCAGCATGCAGCTTGGACAACCAAT

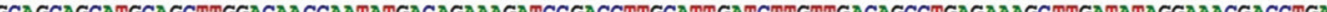
GCAGCAGCATGAGTGGA GCAGCAGAACACACCTTGAACAACCAATATGTCAAAAAGATCCGACCTTGCATTGACCTTGTCGACAACCTGAGAATGCTTGGTATPAGGAAACGACCTGA GCAGCAGAACACA CCTTGCACAA CCAATATGACAAAAAGATCCGACCTTGTATTGACCTCGTTGACAGCCTGAGAATGCTTGGTATAGGAAACGACCTGA GCAGCAGAACACA GCTTGTACAATCAGTATGAGGAGAAGATCCGGCCTTGCATTGATCTCATTGACAGCCTGAGAGCACTTGGAATAGAAAAAGACCTGT GCAGCAGAGCATACCTTGTACAATCAATATGAGGAAAAGATCCGACCCTGTATTGATCTCATCGACAGCTTGAGAGCTCTTGGAATAGAAAAAGACCTGG

$$
\begin{array}{llllllll}
110 & 120 & 130 & 140 & 150 & 160 & 170 & 180
\end{array}
$$

$\ldots|\ldots| \ldots|\ldots| \ldots|\ldots| \ldots|\ldots| \ldots|\ldots| \ldots|\ldots| \ldots|\ldots| \ldots|\ldots| \ldots|\ldots| \ldots|\ldots|$ TGTTGCCTATGAT CACAGTGATTGGAGACCAGAACTCTGGGAAAGTCTGTCCTTGAACTTTGTCTGGTGTTGCTCTTCCTAG TGTTGCCTATGAT CACAGTGATTGGAGACCAGAACTCTGGGAAAAGCTCTGTCCTTGAAGCTTTGTCTGGTGTTGCTCTTCCTAG TGTTGCCTGCAAT CGCAGTGATTGGAGACCGGAACTCTGGGAAAAGCTCTGTCCTTGAAGCTTTGTCTGGTGTTGCTCTTCCTAG TGTTGCCTGCAATCGCAGTGATTGGAGACCGGAACTCTGGGAAAAGCTCTGTCCTTGAAGCTTTGTCTGGTGTTGCTCTTCCTAG TGTTGCCTGCAAT CGCAGTGATTGGAGACCGGAACTCTGGGAAAAGCTCTGTCCTTGAAGCTTTGTCTGGTGTTGCTCTTCCTAG TGTTGCCTGCAATTGCAGTGATTGGAGACCGGAACTCTGGGAAAAGCTCTGTCCTTGAAGCTTT GTCTGGTGTTGCTCTTCCTAG CGTTGCCTGCAATCGCAGTGATTGGAGACCAGAACTCTGGGAAAAGCTCTGTCCTTGAAGCTTTGTCTGGTGITGCTCTTCCTAG CATTGCCTGCAAT CGCAGTGATTGGAGACCAGAGCTCTGGGAAAAGCTCCATCCTCGAAGCCCT GTCTGGTGTTTCTCTTCCTAG CTTTGCCCGCGATCGCAGTGATTGGAGACCAGAGTTCTGGGAAAAGCTCTGTCCTAGAAGCCCT GTCTGGTATTGCTCTTCCTAG

Figure-1: Aligned nucleotide sequence of $185 \mathrm{bp}$ fragment of $M \times 1$ gene.

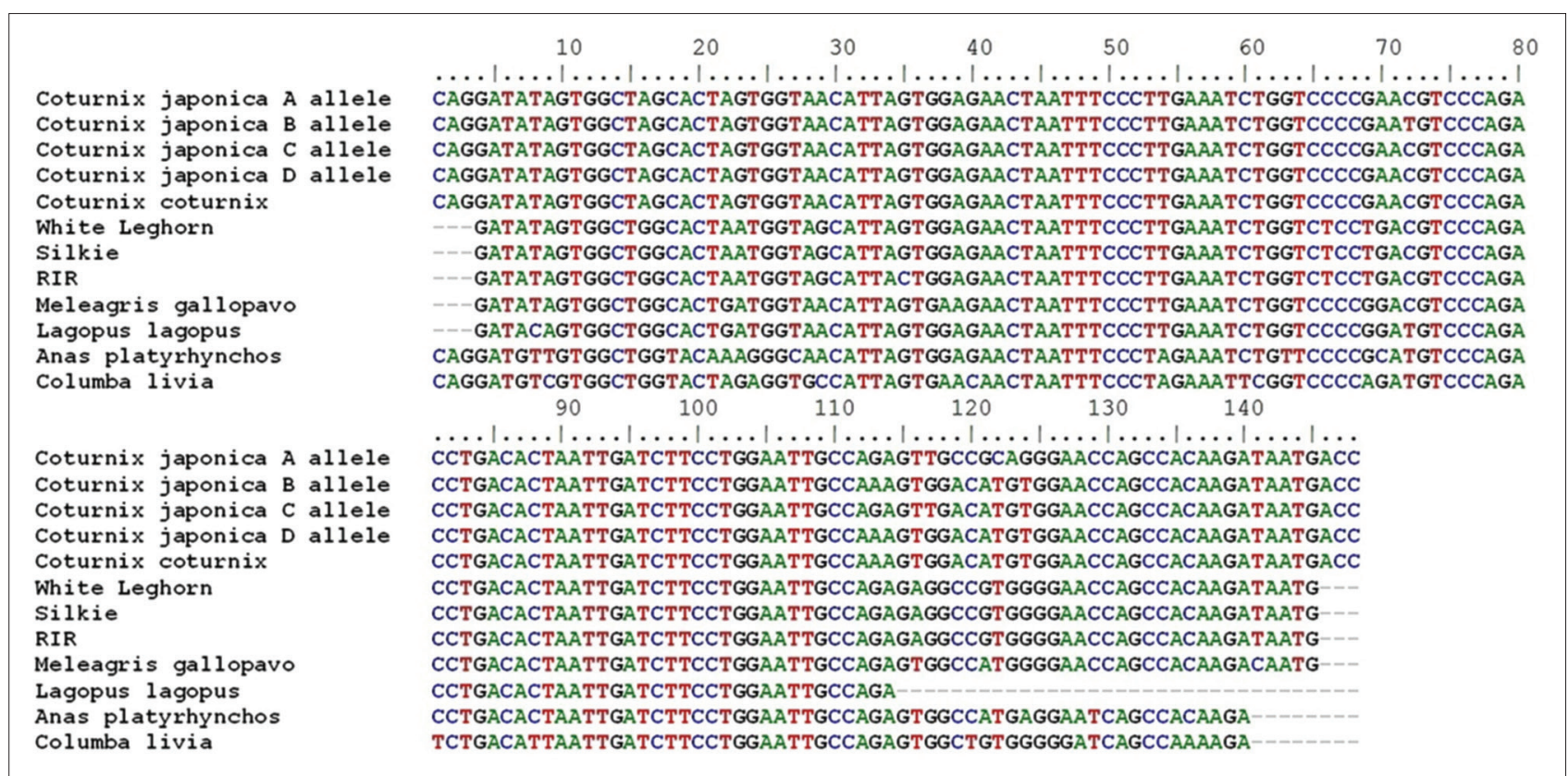

Figure-2: Aligned nucleotide sequence of 148 bp fragment of $M \times 1$ gene.

\begin{tabular}{llc}
\hline & 10 & 20
\end{tabular}

Figure-3: Aligned nucleotide sequence of 161 bp fragment of Mx1 gene.

was identified in Fragment III, and no SNP was identified in fragment No. I and IV. However, between the quail (Japanese quail and common quail) and chicken breeds 94 SNPs were identified, out of which, 10 were species specific (Tables-3-6). In the $5^{\text {th }}$ exon (Fragment II) where four different alleles 
Coturnix japonica Coturnix coturnix White Leghorn Silkie Rhode Island Red Meleagris gallopavo Lagopus lagopus

Phasianus colchicus

Coturnix japonica Coturnix coturnix White Leghorn

Silkie

Rhode Island Red Meleagris gallopavo Lagopus lagopus

Phasianus colchicus
20 30 40 60 70 100 $\ldots|\ldots| \ldots|\ldots| \ldots|\ldots| \ldots|\ldots| \ldots|\ldots| \ldots|\ldots| \ldots|\ldots| \ldots|\ldots| \ldots|\ldots| \ldots|\ldots| \ldots|\ldots| \ldots|\ldots| \ldots|\ldots| \ldots \mid$ GCAAGCAACAGCMGCGAAAATGCATCCAGACTCAATCAAAA

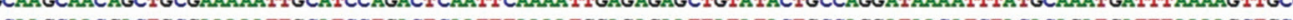
CAAGCAACGACTGCGAAAAATTGCATCCTGACT CAATT TAAAATGGAGAGAATTATATACTGCCAGGATAACATCTACACAGATGATTTAAAAGCTGCC CAAGCAACGACTGCGAAAAATTGCATCCTGACT CAATTTAAAATGGAGAGAATTATATACTGCCAGGATAACATCTACACAGATGATTTAAAAGCTGCC CAAGCAACGACTGCGAAAAATTGCATCCTGACTCAATT TAAAATGGAGAGAATTATATACTGCCAGGATAACATCTACACAGATGATTTAAAAGCTGCC CAAGCAACGACTGGGAAAAATTT CATCCTGACT CAATTTAAAATGGAGAGAATTGTATACTGCCAGGATAACATTTACACAGAT GATTTAAAAGCTGCC CAAGCAACGACTGCGAAAAATTTCATCCTGACT CAATTTAAAATGGAGAAAATTGTA TACTGCCAGGATAAGATTTACGCAGATGATTTAAAAGCTGCC CAAGCAACGACTGCGAAAAACTTCATCCAGACTCAATTTAAAATGGAGAGAACTGTATACTGCCAGGATAACATTTACGCAGATGATTTAAAAGCTGCC

$$
110
$$
120 130 140 150 160 170 180 190 $\ldots|\ldots| \ldots|\ldots| \ldots|\ldots| \ldots|\ldots| \ldots|\ldots| \ldots|\ldots| \ldots|\ldots| \ldots|\ldots| \ldots|\ldots| \ldots|\ldots| \ldots|\ldots| \ldots$ AAGGCAGAAAGCATCAATGCICTMGGITTGGACATGCT AAGGCAGAAAGCATCAATNNNNNNNNNNNNNNNNGCTCITGGIIGAGACATGCINCAAATCAAGGICCCAGCITGICCIGGAAATGGTTI AGGGCAGAAGGCAT CAGCAAAGATACAAAAATCAAAGACCTTGCTITTGGATGTGCTHCACGTCAATGTCCCAGCTITGCCCTGGAAATGGTTT AGGGCAGAAGGCAT CAGCAAAGATACAAAAATCAAAGACCTTGCTTTTGGATGTGCTTCACGTCAATGTCCCAGCTTTGCCCTGGAAATGGTTT AGGGCAGAAGGCATCAGCAAAGATACAAAAATCAAAGACCTTGCTTTTGGATGTGCTTCACGTCAATGTCCCAGCTTTGCCCTGGAAATGGTTT AGGGCAGAAAGCAT CAACAAAAATACAAAAATCAAAGACCTTGATCCTGGAATTGCTT CAAGCAAAGGTCCCAGCTTTGTCCTGGAAATGGTTT CGGGCAGAAAGCATCAACAAAAATACAAAAATCAAAGACCTTGGTCCTGCAATTGCTTCAAGTCAAGGTTCCAGCTTTGTCTTGGAAATGGTTT AGGGCAGAAAACATCAACAAAAATACAAAAATCAAAGACCTTAGTCCTAAAATTGCTTCAAGTCAAGGTCCCAGCTTTGTCCTGGAAATGGTTT

Figure-4: Aligned nucleotide sequence of $176 \mathrm{bp}$ fragment of $M \times 1$ gene.

Table-3: Nucleotide substitutions in Fragment I (Exon 3 of Japanese quail Mx1 gene).

\begin{tabular}{|c|c|c|c|c|c|c|c|c|c|}
\hline $\begin{array}{l}\text { Position } \\
\text { (bp) }\end{array}$ & $\begin{array}{l}\text { Coturnix } \\
\text { japonica }\end{array}$ & $\begin{array}{l}\text { Coturnix } \\
\text { coturnix }\end{array}$ & WLH & Silki & RIR & Turkey & $\begin{array}{l}\text { Willow } \\
\text { ptarmigan }\end{array}$ & Duck & Pigeon \\
\hline $\begin{array}{l}8 \\
9 \\
10 \\
11 \\
12 \\
14 \\
18 \\
19 \\
24 \\
25 \\
27 \\
32 \\
33 \\
34 \\
35 \\
36 \\
42 \\
45 \\
48 \\
51 \\
57 \\
60 \\
61 \\
63 \\
66 \\
68 \\
70 \\
76 \\
77 \\
78 \\
83 \\
84 \\
89 \\
93 \\
94 \\
96 \\
100 \\
101 \\
102 \\
108 \\
109 \\
110 \\
111 \\
114 \\
115 \\
131 \\
134 \\
135 \\
150 \\
151 \\
156 \\
162 \\
163 \\
165 \\
171 \\
172 \\
175 \\
182\end{array}$ & 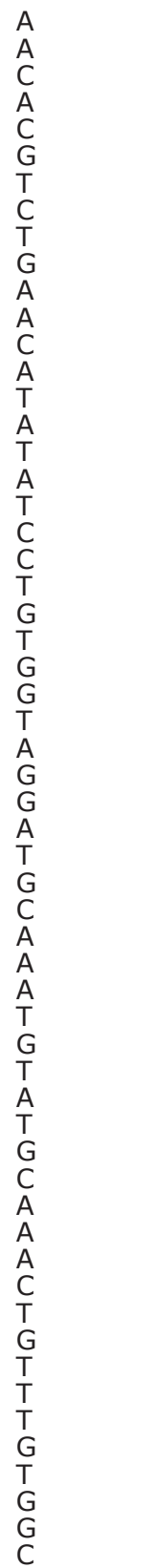 & 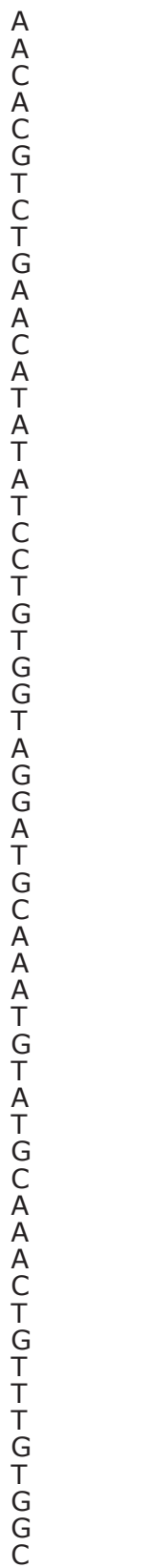 & 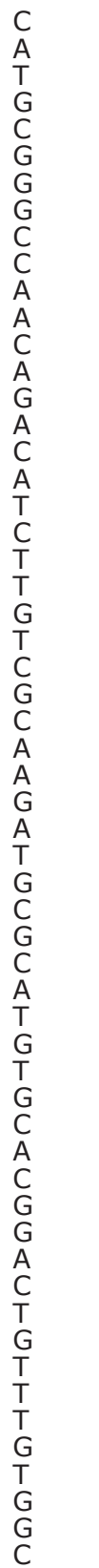 & $\begin{array}{l}C \\
A \\
T \\
G \\
C \\
G \\
G \\
G \\
C \\
C \\
C \\
A \\
A \\
C \\
A \\
G \\
A \\
A \\
C \\
A \\
T \\
C \\
T \\
T \\
G \\
T \\
C \\
G \\
G \\
C \\
A \\
A \\
G \\
A \\
A \\
T \\
G \\
C \\
G\end{array}$ & 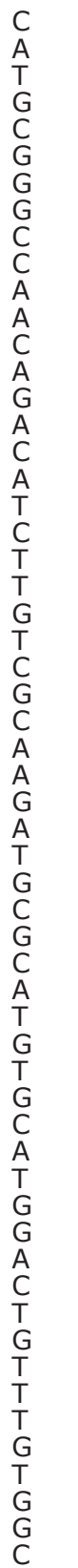 & $\begin{array}{l}A \\
A \\
C \\
A \\
C \\
C \\
C \\
G \\
A \\
C \\
C \\
A \\
A \\
G \\
C \\
A \\
A \\
A \\
A \\
C \\
A \\
T \\
C \\
T \\
T \\
G \\
C \\
C \\
C \\
A \\
C \\
A \\
T \\
G \\
G \\
G \\
T \\
G \\
C \\
G \\
C \\
C \\
A \\
C \\
G\end{array}$ & 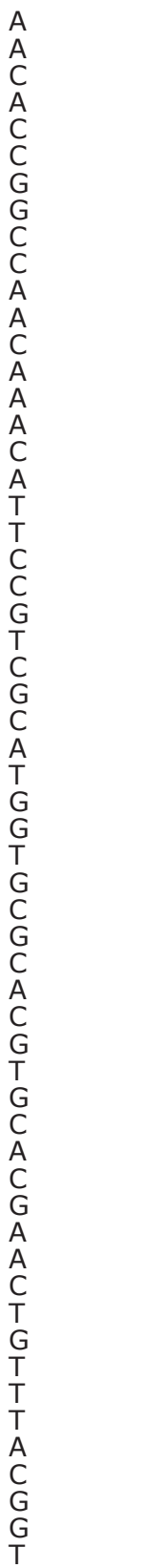 & 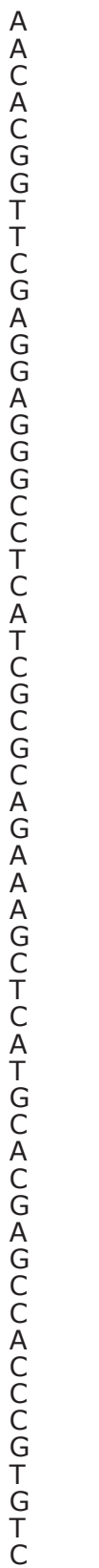 & $\begin{array}{l}A \\
G \\
C \\
A \\
T \\
C \\
C \\
G \\
T \\
T \\
C \\
A \\
A \\
G \\
G \\
G \\
A \\
A \\
C \\
A \\
T \\
T \\
C \\
C \\
A \\
A \\
C \\
C \\
G \\
T \\
G \\
C \\
T \\
G \\
A \\
A \\
A \\
A \\
G\end{array}$ \\
\hline
\end{tabular}


Table-4: Nucleotide substitutions in Fragment II (Partial exon 5 of Japanese quail $M \times 1$ gene).

\begin{tabular}{|c|c|c|c|c|c|c|c|c|c|c|c|c|}
\hline \multirow{2}{*}{$\begin{array}{l}\text { Position } \\
\text { (bp) }\end{array}$} & \multicolumn{4}{|c|}{ Coturnix japonica } & \multirow{2}{*}{$\begin{array}{l}\text { Coturnix } \\
\text { coturnix }\end{array}$} & \multirow[t]{2}{*}{ WLH } & \multirow[t]{2}{*}{ Silki } & \multirow[t]{2}{*}{ RIR } & \multirow[t]{2}{*}{ Turkey } & \multirow{2}{*}{$\begin{array}{l}\text { Willow } \\
\text { ptarmigan }\end{array}$} & \multirow[t]{2}{*}{ Duck } & \multirow[t]{2}{*}{ Pigeon } \\
\hline & A Allele & B Allele & C Allele & D Allele & & & & & & & & \\
\hline 7 & $A$ & $A$ & $A$ & $A$ & $A$ & $A$ & $A$ & $A$ & $A$ & $A$ & $\mathrm{G}$ & $\mathrm{G}$ \\
\hline 8 & $\mathrm{~T}$ & $\mathrm{~T}$ & $\mathrm{~T}$ & $\mathrm{~T}$ & $\mathrm{~T}$ & $\mathrm{~T}$ & $\mathrm{~T}$ & $\mathrm{~T}$ & $\mathrm{~T}$ & $\mathrm{C}$ & $\mathrm{T}$ & $\mathrm{T}$ \\
\hline 9 & $A$ & $A$ & $A$ & $A$ & $A$ & $A$ & $A$ & $A$ & $A$ & $A$ & $\mathrm{~T}$ & $\mathrm{~T}$ \\
\hline 16 & $A$ & $A$ & $A$ & $A$ & G & G & G & G & G & G & G & G \\
\hline 18 & C & $\mathrm{C}$ & $\mathrm{C}$ & $\mathrm{C}$ & $\mathrm{C}$ & $\mathrm{C}$ & $\mathrm{C}$ & $\mathrm{C}$ & $\mathrm{C}$ & $\mathrm{C}$ & $\mathrm{T}$ & $\mathrm{T}$ \\
\hline 21 & $\mathrm{~T}$ & $\mathrm{~T}$ & $\mathrm{~T}$ & $\mathrm{~T}$ & $\mathrm{~T}$ & $\mathrm{~T}$ & $\mathrm{~T}$ & $\mathrm{~T}$ & $\mathrm{~T}$ & $\mathrm{~T}$ & $A$ & $\mathrm{~T}$ \\
\hline 22 & $A$ & $A$ & $A$ & $A$ & $A$ & $A$ & $A$ & $A$ & $\mathrm{G}$ & G & $A$ & $A$ \\
\hline 23 & $\mathrm{G}$ & $\mathrm{G}$ & $\mathrm{G}$ & $\mathrm{G}$ & $A$ & $A$ & $A$ & $A$ & $\mathrm{G}$ & $A$ & $A$ & G \\
\hline 24 & $\mathrm{~T}$ & $\mathrm{~T}$ & $\mathrm{~T}$ & $\mathrm{~T}$ & $\mathrm{~T}$ & $\mathrm{~T}$ & $\mathrm{~T}$ & $\mathrm{~T}$ & $\mathrm{~T}$ & $\mathrm{~T}$ & G & $A$ \\
\hline 27 & $\mathrm{~T}$ & $\mathrm{~T}$ & $\mathrm{~T}$ & $\mathrm{~T}$ & $\mathrm{~T}$ & $\mathrm{~T}$ & $\mathrm{~T}$ & $\mathrm{~T}$ & $T$ & $\mathrm{~T}$ & C & $\mathrm{T}$ \\
\hline 28 & $A$ & $A$ & $A$ & $A$ & $A$ & $A$ & $A$ & $A$ & $A$ & $A$ & $A$ & G \\
\hline 29 & $A$ & $A$ & $A$ & $A$ & $A$ & G & $\mathrm{G}$ & G & $A$ & $A$ & $A$ & C \\
\hline 35 & G & G & $\mathrm{G}$ & G & $\mathrm{G}$ & $\mathrm{G}$ & G & C & $\mathrm{G}$ & $\mathrm{G}$ & G & $\mathrm{G}$ \\
\hline 38 & G & G & $\mathrm{G}$ & G & G & G & G & G & $A$ & G & G & $A$ \\
\hline 40 & G & G & $\mathrm{G}$ & G & G & G & $\mathrm{G}$ & G & $\mathrm{G}$ & G & G & C \\
\hline 54 & $\mathrm{~T}$ & $\mathrm{~T}$ & $\mathrm{~T}$ & $\mathrm{~T}$ & $\mathrm{~T}$ & $\mathrm{~T}$ & $\mathrm{~T}$ & $\mathrm{~T}$ & $\mathrm{~T}$ & $\mathrm{~T}$ & A & $A$ \\
\hline 60 & C & C & $\mathrm{C}$ & C & C & C & C & C & $\mathrm{C}$ & C & C & $\mathrm{T}$ \\
\hline 61 & $\mathrm{~T}$ & $\mathrm{~T}$ & $\mathrm{~T}$ & $\mathrm{~T}$ & $\mathrm{~T}$ & $\mathrm{~T}$ & $\mathrm{~T}$ & $\mathrm{~T}$ & $\mathrm{~T}$ & $\mathrm{~T}$ & $\mathrm{~T}$ & C \\
\hline 63 & G & G & $\mathrm{G}$ & G & G & G & G & G & G & G & $\mathrm{T}$ & G \\
\hline 66 & C & C & C & C & C & $\mathrm{T}$ & $\mathrm{T}$ & $\mathrm{T}$ & C & C & C & C \\
\hline 69 & $\mathrm{G}$ & G & $\mathrm{G}$ & G & G & $\mathrm{T}$ & $\mathrm{T}$ & $\mathrm{T}$ & G & G & G & $\mathrm{G}$ \\
\hline 70 & $A$ & $A$ & $A$ & $A$ & $A$ & $\mathrm{G}$ & G & G & $\mathrm{G}$ & G & C & G \\
\hline 72 & C & $\mathrm{T}$ & C & C & C & C & C & C & C & $\mathrm{T}$ & $\mathrm{T}$ & $\mathrm{T}$ \\
\hline 81 & C & C & C & C & C & C & C & C & C & C & C & $\mathrm{T}$ \\
\hline 88 & C & C & C & C & C & C & C & C & C & C & C & $\mathrm{T}$ \\
\hline 113 & G & $A$ & $\mathrm{G}$ & $A$ & $A$ & $\mathrm{G}$ & $\mathrm{G}$ & G & $\mathrm{G}$ & $\mathrm{G}$ & $\mathrm{G}$ & G \\
\hline 116 & $\mathrm{~T}$ & $\mathrm{~T}$ & $\mathrm{~T}$ & $\mathrm{~T}$ & $\mathrm{~T}$ & $A$ & $A$ & $A$ & $\mathrm{~T}$ & - & $\mathrm{T}$ & $\mathrm{T}$ \\
\hline 117 & $\mathrm{~T}$ & $\mathrm{G}$ & $\mathrm{T}$ & $\mathrm{G}$ & G & G & $\mathrm{G}$ & $\mathrm{G}$ & $\mathrm{G}$ & - & G & G \\
\hline 119 & C & $A$ & $A$ & $A$ & $A$ & $\mathrm{C}$ & C & C & C & - & $\mathrm{C}$ & C \\
\hline 121 & G & $A$ & $A$ & $A$ & $A$ & G & $\mathrm{G}$ & G & $A$ & - & $A$ & $\mathrm{G}$ \\
\hline 122 & C & $\mathrm{T}$ & $\mathrm{T}$ & $\mathrm{T}$ & $\mathrm{T}$ & $\mathrm{T}$ & $\mathrm{T}$ & $\mathrm{T}$ & $\mathrm{T}$ & - & $\mathrm{T}$ & $\mathrm{T}$ \\
\hline 123 & $A$ & G & $\mathrm{G}$ & $\mathrm{G}$ & G & G & G & G & G & - & G & $\mathrm{G}$ \\
\hline 124 & G & $\mathrm{T}$ & $\mathrm{T}$ & $\mathrm{T}$ & $\mathrm{T}$ & $\mathrm{G}$ & $\mathrm{G}$ & G & $\mathrm{G}$ & - & $A$ & G \\
\hline 127 & $A$ & $A$ & $A$ & $A$ & $A$ & $A$ & $A$ & $A$ & $A$ & - & G & G \\
\hline 129 & C & C & C & C & C & C & C & C & C & - & $\mathrm{T}$ & $\mathrm{T}$ \\
\hline 136 & C & C & $\mathrm{C}$ & C & $\mathrm{C}$ & C & C & C & C & - & C & $A$ \\
\hline 141 & $\mathrm{~T}$ & $\mathrm{~T}$ & $\mathrm{~T}$ & $\mathrm{~T}$ & $\mathrm{~T}$ & $\mathrm{~T}$ & $\mathrm{~T}$ & $\mathrm{~T}$ & C & - & - & - \\
\hline
\end{tabular}

\begin{tabular}{|l|c|c|c|c|c|c|c|c|c|}
\hline & 1 & 2 & 3 & 4 & 5 & 6 & 7 & 8 & 9 \\
\cline { 2 - 12 } & & 0.000 & 0.049 & 0.049 & 0.049 & 0.044 & 0.041 & 0.082 & 0.076 \\
1. Coturnix japonica & & & 0.049 & 0.049 & 0.049 & 0.044 & 0.041 & 0.082 & 0.076 \\
2. Coturnix coturnix & 0.000 & & & 0.000 & 0.000 & 0.024 & 0.023 & 0.060 & 0.066 \\
3. White Leghorn & 0.124 & 0.124 & & 0.000 & 0.024 & 0.023 & 0.060 & 0.066 \\
4. Silkie & 0.124 & 0.124 & 0.000 & & 0.000 & 0.060 & 0.066 \\
\hline 5. RIR & 0.124 & 0.124 & 0.000 & 0.000 & & 0.024 & 0.023 & 0.060 \\
6. Meleagris gallopavo & 0.105 & 0.105 & 0.033 & 0.033 & 0.033 & & 0.016 & 0.055 & 0.061 \\
7. Lagopus lagopus & 0.087 & 0.087 & 0.033 & 0.033 & 0.033 & 0.016 & & 0.056 & 0.063 \\
8. Anas platyrhynchos & 0.257 & 0.257 & 0.164 & 0.164 & 0.164 & 0.143 & 0.145 & & 0.039 \\
9. Columba livia & 0.215 & 0.215 & 0.168 & 0.168 & 0.168 & 0.147 & 0.149 & 0.086 & \\
\hline
\end{tabular}

Figure-5: Nucleotide sequence distance of 185 bp fragment of $M x 1$ gene between different species (The number of base substitutions per site between sequences is shown. Standard error estimate(s) are shown above the diagonal and were obtained by a bootstrap procedure 1000 replicates. Analyses were conducted using the Kimura 2-parameter model).

were identified) was found to be highly polymorphic and most of its nucleotide substitutions are non-synonymous. The allele "A" of $5^{\text {th }}$ exon of Fragment II in Japanese quail appeared to have maximum nucleotide substitutions as compared to other three alleles.

Sequence divergence analysis using MEGA 6 with 1000 replicates of bootstrap and Kimura 2 parameter model revealed that sequence of Japanese quail is almost
$100 \%$ identical to that of common quail (Figures-5-8) in all the four fragments. However, the sequence of Japanese quail showed divergence of $12.4 \%, 5.7 \%$, $10.1 \%$, and $17.7 \%$ from sequence of chicken as well as $10.5 \%, 8.8 \%, 8.0 \%$, and $17.7 \%$ from sequence of turkey in Fragment I, II, III, and IV, respectively (Figures-5-8).

The phylogenetic tree analysis of the amplified sequence of four fragments revealed that Japanese 
Table-5: Nucleotide substitutions in Fragment III (partial exon 7 of Japanese quail $M x 1$ gene).

\begin{tabular}{|c|c|c|c|c|c|c|c|c|}
\hline Position & Coturnix japonica & Coturnix coturnix & WLH & Silki & RIR & Turkey & Willow ptarmigan & Pheasant \\
\hline 5 & $\mathrm{C}$ & $\mathrm{C}$ & $\mathrm{C}$ & $\mathrm{C}$ & $\mathrm{C}$ & $\mathrm{C}$ & $\mathrm{T}$ & C \\
\hline 8 & $\mathrm{~T}$ & $\mathrm{~T}$ & $\mathrm{~T}$ & $\mathrm{~T}$ & $\mathrm{~T}$ & C & $\mathrm{T}$ & $\mathrm{T}$ \\
\hline 17 & $\mathrm{~T}$ & $\mathrm{~T}$ & C & C & C & $\mathrm{T}$ & $\mathrm{T}$ & $\mathrm{T}$ \\
\hline 20 & G & G & $A$ & $A$ & $A$ & G & G & G \\
\hline 24 & G & G & $A$ & $A$ & $A$ & G & G & G \\
\hline 26 & $\mathrm{C}$ & $\mathrm{C}$ & $\mathrm{C}$ & $\mathrm{C}$ & $\mathrm{C}$ & $\mathrm{T}$ & $\mathrm{C}$ & $\mathrm{C}$ \\
\hline 27 & $\mathrm{C}$ & $\mathrm{C}$ & G & G & G & $A$ & $A$ & G \\
\hline 29 & $\mathrm{C}$ & $\mathrm{C}$ & $A$ & $A$ & $A$ & $A$ & $A$ & $A$ \\
\hline 30 & $A$ & $A$ & G & G & G & G & G & G \\
\hline 40 & G & G & $A$ & $A$ & $A$ & $A$ & $A$ & $A$ \\
\hline 42 & $A$ & $A$ & $A$ & $A$ & $A$ & G & G & G \\
\hline 47 & C & C & C & C & C & $\mathrm{T}$ & C & C \\
\hline 52 & G & $\mathrm{G}$ & $A$ & $A$ & $A$ & $A$ & $A$ & $A$ \\
\hline 57 & $A$ & A & G & G & $\mathrm{G}$ & $A$ & $A$ & $A$ \\
\hline 59 & G & $\mathrm{G}$ & $A$ & $A$ & A & G & G & G \\
\hline 61 & $A$ & $A$ & $A$ & $A$ & A & G & G & $A$ \\
\hline 65 & $\mathrm{~T}$ & $\mathrm{~T}$ & C & $\mathrm{C}$ & $\mathrm{C}$ & $\mathrm{T}$ & $\mathrm{T}$ & $\mathrm{T}$ \\
\hline 68 & G & G & G & G & G & $A$ & $A$ & $A$ \\
\hline 72 & G & G & A & A & A & A & A & A \\
\hline 74 & $\mathrm{~T}$ & $\mathrm{~T}$ & C & C & C & C & C & C \\
\hline 104 & G & G & A & A & A & G & G & G \\
\hline 108 & C & C & $\mathrm{T}$ & $\mathrm{T}$ & $\mathrm{T}$ & C & C & C \\
\hline 110 & C & C & $\mathrm{T}$ & $\mathrm{T}$ & $\mathrm{T}$ & C & $\mathrm{T}$ & C \\
\hline 117 & C & C & A & A & A & C & C & C \\
\hline 118 & A & A & $\mathrm{T}$ & $\mathrm{T}$ & $\mathrm{T}$ & A & A & A \\
\hline 122 & $\mathrm{~T}$ & $\mathrm{~T}$ & C & C & C & C & C & C \\
\hline 123 & G & G & $\mathrm{T}$ & $\mathrm{T}$ & $\mathrm{T}$ & A & A & A \\
\hline 126 & A & A & $\mathrm{T}$ & $\mathrm{T}$ & $\mathrm{T}$ & A & A & A \\
\hline 127 & A & A & G & G & G & A & A & A \\
\hline 128 & C & C & C & C & C & C & $\mathrm{T}$ & $\mathrm{T}$ \\
\hline 129 & A & A & A & A & A & G & G & G \\
\hline 132 & G & G & G & G & G & A & A & A \\
\hline 133 & A & A & A & A & A & A & A & G \\
\hline 134 & A & A & A & A & A & $\mathrm{T}$ & $\mathrm{T}$ & $\mathrm{T}$ \\
\hline 135 & $\mathrm{~T}$ & $\mathrm{~T}$ & $\mathrm{~T}$ & $\mathrm{~T}$ & $\mathrm{~T}$ & C & C & C \\
\hline 143 & G & G & C & C & C & G & G & G \\
\hline 144 & G & $\mathrm{G}$ & $A$ & $A$ & A & G & G & G \\
\hline 149 & G & $\mathrm{G}$ & C & $\mathrm{C}$ & C & G & G & G \\
\hline 150 & G & G & $A$ & $A$ & $\mathrm{G}$ & G & G & G \\
\hline 151 & $\mathrm{~T}$ & $\mathrm{~T}$ & C & C & C & C & C & C \\
\hline
\end{tabular}

\begin{tabular}{|l|c|c|c|c|c|c|c|c|c|c|c|c|}
\hline & 1 & 2 & 3 & 4 & 5 & 6 & 7 & 8 & 9 & 10 & 11 & 12 \\
\hline 1. Coturnix japonica A allele & & 0.000 & 0.000 & 0.000 & 0.000 & 0.041 & 0.041 & 0.041 & 0.054 & 0.054 & 0.052 & 0.107 \\
2. Coturnix japonica B allele & 0.000 & & 0.000 & 0.000 & 0.000 & 0.041 & 0.041 & 0.041 & 0.054 & 0.054 & 0.052 & 0.107 \\
3. Coturnix japonica C allele & 0.000 & 0.000 & & 0.000 & 0.000 & 0.041 & 0.041 & 0.041 & 0.054 & 0.054 & 0.052 & 0.107 \\
4. Coturnix japonica D allele & 0.000 & 0.000 & 0.000 & & 0.000 & 0.041 & 0.041 & 0.041 & 0.054 & 0.054 & 0.052 & 0.107 \\
5. Coturnix coturnix & 0.000 & 0.000 & 0.000 & 0.000 & & 0.041 & 0.041 & 0.041 & 0.054 & 0.054 & 0.052 & 0.107 \\
5. White Leghorn & 0.057 & 0.057 & 0.057 & 0.057 & 0.057 & & 0.000 & 0.000 & 0.029 & 0.029 & 0.040 & 0.080 \\
7. Silkie & 0.057 & 0.057 & 0.057 & 0.057 & 0.057 & 0.000 & & 0.000 & 0.029 & 0.029 & 0.040 & 0.080 \\
3. RIR & 0.057 & 0.057 & 0.057 & 0.057 & 0.057 & 0.000 & 0.000 & & 0.029 & 0.029 & 0.040 & 0.080 \\
9. Meleagris gallopavo & 0.088 & 0.088 & 0.088 & 0.088 & 0.088 & 0.028 & 0.028 & 0.028 & & 0.000 & 0.053 & 0.092 \\
10. Lagopus lagopus & 0.088 & 0.088 & 0.088 & 0.088 & 0.088 & 0.028 & 0.028 & 0.028 & 0.000 & & 0.053 & 0.092 \\
11. Anas platyrhynchos & 0.086 & 0.086 & 0.086 & 0.086 & 0.086 & 0.056 & 0.056 & 0.056 & 0.086 & 0.086 & & 0.073 \\
12. Columba livia & 0.230 & 0.230 & 0.230 & 0.230 & 0.230 & 0.153 & 0.153 & 0.153 & 0.190 & 0.190 & 0.150 & \\
\hline
\end{tabular}

Figure-6: Nucleotide sequence distance of 148 bp fragment of $M x 1$ gene between different species (The number of base substitutions per site between sequences is shown. Standard error estimate(s) are shown above the diagonal and were obtained by a bootstrap procedure 1000 replicates. Analyses were conducted using the Kimura 2-parameter model).

quail and common quail always remains in the same cluster indicating their common ancestral origin (Figures-9-12). The deduced amino acid sequences from the nucleotide sequences of four fragments from Japanese quail, common quail, and chicken were analyzed for sequence homology. Within quail sequences, only four amino acid substitutions were observed (present in Fragment II only). However, between quail and chicken, $56(12+10+14+20)$ amino acid substitutions were identified. Clustering of chicken sequences in one cluster along with grouping of common quail and Japanese quail in other cluster is very well expected as per taxonomic classification keeping Japanese quail (Coturnix Japonica) and common quail (Coturnix coturnix) in the one genus Coturnix, whereas 
Table-6: Nucleotide substitutions in Fragment IV (partial exon 13 of Japanese quail $M \times 1$ gene).

\begin{tabular}{|c|c|c|c|c|c|c|c|c|}
\hline Position & Coturnix japonica & Coturnix coturnix & WLH & Silki & RIR & Turkey & Willow ptarmigan & Pheasant \\
\hline 10 & $A$ & $A$ & $\mathrm{G}$ & $\mathrm{G}$ & G & $\mathrm{G}$ & $\mathrm{G}$ & $\mathrm{G}$ \\
\hline 11 & G & G & $A$ & $A$ & $A$ & $A$ & $A$ & $A$ \\
\hline 15 & $\mathrm{C}$ & $\mathrm{C}$ & $\mathrm{C}$ & $\mathrm{C}$ & $\mathrm{C}$ & G & $\mathrm{C}$ & $\mathrm{C}$ \\
\hline 22 & $\mathrm{~T}$ & $\mathrm{~T}$ & $\mathrm{~T}$ & $\mathrm{~T}$ & $\mathrm{~T}$ & $\mathrm{~T}$ & $\mathrm{~T}$ & $\mathrm{C}$ \\
\hline 24 & G & G & G & G & G & $\mathrm{T}$ & $\mathrm{T}$ & $\mathrm{T}$ \\
\hline 30 & $A$ & $A$ & $\mathrm{~T}$ & $\mathrm{~T}$ & $\mathrm{~T}$ & $\mathrm{~T}$ & $\mathrm{~T}$ & $\mathrm{~T}$ \\
\hline 40 & $\mathrm{C}$ & $\mathrm{C}$ & $\mathrm{T}$ & $\mathrm{T}$ & $\mathrm{T}$ & $\mathrm{T}$ & $\mathrm{T}$ & $\mathrm{T}$ \\
\hline 46 & $\mathrm{~T}$ & $\mathrm{~T}$ & G & G & G & G & G & G \\
\hline 51 & G & $\mathrm{G}$ & $\mathrm{G}$ & $\mathrm{G}$ & $\mathrm{G}$ & $\mathrm{G}$ & $A$ & $\mathrm{G}$ \\
\hline 53 & $\mathrm{G}$ & $\mathrm{G}$ & $A$ & $A$ & $A$ & $A$ & $A$ & $A$ \\
\hline 54 & $\mathrm{C}$ & $\mathrm{C}$ & $\mathrm{T}$ & $T$ & $\mathrm{~T}$ & $\mathrm{~T}$ & $\mathrm{~T}$ & C \\
\hline 56 & $\mathrm{G}$ & $\mathrm{G}$ & $A$ & $A$ & $A$ & $\mathrm{G}$ & $\mathrm{G}$ & $\mathrm{G}$ \\
\hline 73 & $A$ & $A$ & C & C & C & C & $\mathrm{G}$ & $\mathrm{C}$ \\
\hline 76 & $\mathrm{~T}$ & $\mathrm{~T}$ & C & C & C & $\mathrm{T}$ & $\mathrm{T}$ & $\mathrm{T}$ \\
\hline 79 & $\mathrm{~T}$ & $\mathrm{~T}$ & C & $\mathrm{C}$ & $\mathrm{C}$ & C & C & C \\
\hline 80 & G & $\mathrm{G}$ & $A$ & $A$ & $A$ & $A$ & $\mathrm{G}$ & $\mathrm{G}$ \\
\hline 83 & $A$ & $A$ & G & $\mathrm{G}$ & $\mathrm{G}$ & $\mathrm{G}$ & $\mathrm{G}$ & G \\
\hline 96 & $\mathrm{~T}$ & $\mathrm{~T}$ & C & C & $\mathrm{C}$ & C & C & C \\
\hline 101 & $A$ & $A$ & $A$ & $A$ & $A$ & $A$ & C & $A$ \\
\hline 102 & $A$ & $A$ & G & $\mathrm{G}$ & $\mathrm{G}$ & $\mathrm{G}$ & $\mathrm{G}$ & $\mathrm{G}$ \\
\hline 110 & $A$ & $A$ & $\mathrm{G}$ & $\mathrm{G}$ & $\mathrm{G}$ & $A$ & $A$ & $A$ \\
\hline 111 & $\mathrm{G}$ & $\mathrm{G}$ & G & $\mathrm{G}$ & $\mathrm{G}$ & $\mathrm{G}$ & $\mathrm{G}$ & $A$ \\
\hline 117 & $A$ & $A$ & G & $\mathrm{G}$ & $\mathrm{G}$ & $A$ & $A$ & $A$ \\
\hline 118 & $\mathrm{~T}$ & $\mathrm{~T}$ & C & C & C & C & C & C \\
\hline 161 & $A$ & A & C & C & C & $A$ & $A$ & $A$ \\
\hline 162 & $A$ & A & G & $\mathrm{G}$ & $\mathrm{G}$ & $\mathrm{G}$ & $\mathrm{G}$ & G \\
\hline 163 & $\mathrm{~T}$ & $\mathrm{~T}$ & $\mathrm{~T}$ & $\mathrm{~T}$ & $\mathrm{~T}$ & C & $\mathrm{T}$ & $\mathrm{T}$ \\
\hline 164 & C & C & C & C & C & $A$ & C & C \\
\hline 167 & $\mathrm{G}$ & G & $\mathrm{T}$ & $\mathrm{T}$ & $\mathrm{T}$ & G & $\mathrm{G}$ & $\mathrm{G}$ \\
\hline 170 & C & C & C & C & C & C & $\mathrm{T}$ & C \\
\hline 180 & $\mathrm{C}$ & $\mathrm{T}$ & C & C & C & $\mathrm{T}$ & $\mathrm{T}$ & $\mathrm{T}$ \\
\hline 182 & C & C & C & C & C & C & $\mathrm{T}$ & C \\
\hline
\end{tabular}

\begin{tabular}{|c|c|c|c|c|c|c|c|c|}
\hline & 1 & 2 & 3 & 4 & 5 & 6 & 7 & 8 \\
\hline 1. Coturnix japonica & & 0.000 & 0.047 & 0.047 & 0.047 & 0.042 & 0.042 & 0.042 \\
\hline 2. Coturnix coturnix & 0.000 & & 0.047 & 0.047 & 0.047 & 0.042 & 0.042 & 0.042 \\
\hline 3. White Leghorn & 0.101 & 0.101 & & 0.000 & 0.000 & 0.034 & 0.034 & 0.034 \\
\hline 4. Silkie & 0.101 & 0.101 & 0.000 & & 0.000 & 0.034 & 0.034 & 0.034 \\
\hline 5. Rhode Island Red & 0.101 & 0.101 & 0.000 & 0.000 & & 0.034 & 0.034 & 0.034 \\
\hline 6. Meleagris gallopavo & 0.080 & 0.080 & 0.058 & 0.058 & 0.058 & & 0.000 & 0.028 \\
\hline 7. Lagopus lagopus & 0.080 & 0.080 & 0.058 & 0.058 & 0.058 & 0.000 & & 0.028 \\
\hline 8. Phasianus colchicus & 0.080 & 0.080 & 0.058 & 0.058 & 0.058 & 0.038 & 0.038 & \\
\hline
\end{tabular}

Figure-7: Nucleotide sequence distance of $161 \mathrm{bp}$ fragment of $M \times 1$ gene between different species (The number of base substitutions per site between sequences is shown. Standard error estimate(s) are shown above the diagonal and were obtained by a bootstrap procedure, 1000 replicates. Analyses were conducted using the Kimura 2-parameter model).

\begin{tabular}{|l|c|c|c|c|c|c|c|c|}
\hline & 1 & 2 & 3 & 4 & 5 & 6 & 7 & 8 \\
\hline 1. Coturnix japonica & & 0.000 & 0.062 & 0.062 & 0.062 & 0.061 & 0.058 & 0.062 \\
\cline { 2 - 10 } 2. Coturnix coturnix & 0.000 & & 0.062 & 0.062 & 0.062 & 0.061 & 0.058 & 0.062 \\
3. White Leghorn & 0.177 & 0.177 & & 0.000 & 0.000 & 0.026 & 0.025 & 0.028 \\
\hline 4. Silkie & 0.177 & 0.177 & 0.000 & & 0.000 & 0.026 & 0.025 & 0.028 \\
5. Rhode Island Red & 0.177 & 0.177 & 0.000 & 0.000 & & 0.026 & 0.025 & 0.028 \\
6. Meleagris gallopavo & 0.177 & 0.177 & 0.036 & 0.036 & 0.036 & & 0.024 & 0.026 \\
7. Lagopus lagopus & 0.156 & 0.156 & 0.035 & 0.035 & 0.035 & 0.035 & & 0.024 \\
8. Phasianus colchicus & 0.177 & 0.177 & 0.036 & 0.036 & 0.036 & 0.036 & 0.035 & \\
\hline
\end{tabular}

Figure-8: Nucleotide sequence distance of 176 bp fragment of $M x 1$ gene between different species (The number of base substitutions a site between sequences is shown. Standard error estimate(s) are shown above the diagonal and were obtained by a bootstrap procedure, 1000 replicates. Analyses were conducted using the Kimura 2-parameter model). 


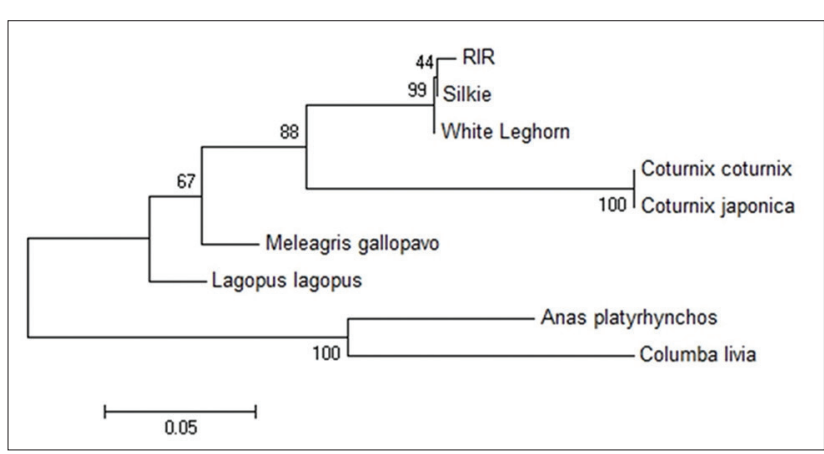

Figure-9: Phylogenetic tree based on 185 bp fragment nucleotide sequence of $M x 1$ gene.

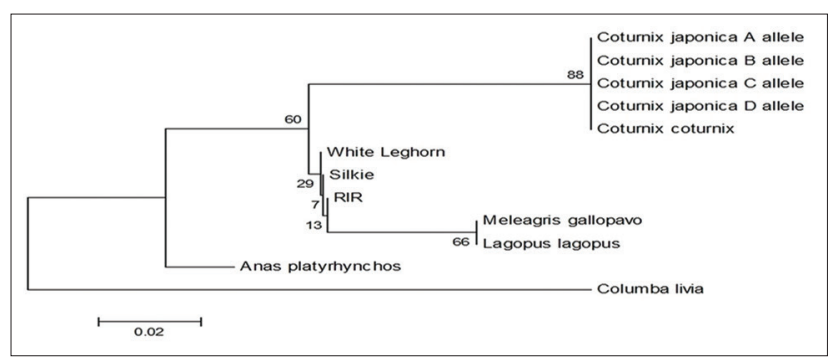

Figure-10: Phylogenetic tree based on 148 bp fragment nucleotide sequence of $M x 1$ gene.

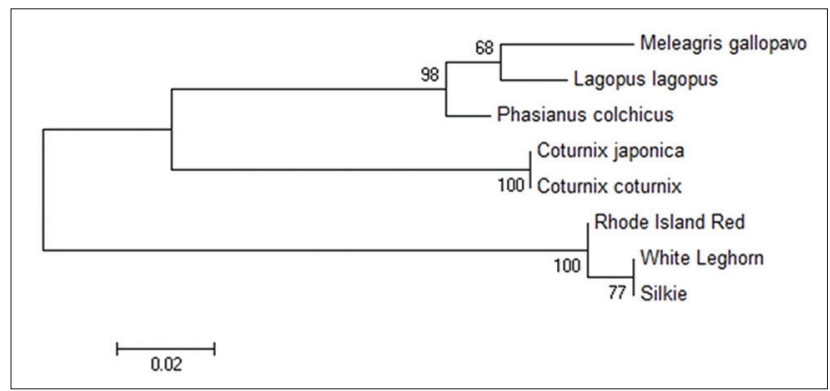

Figure-11: Phylogenetic tree based on 161 bp fragment nucleotide sequence of $M \times 1$ gene.

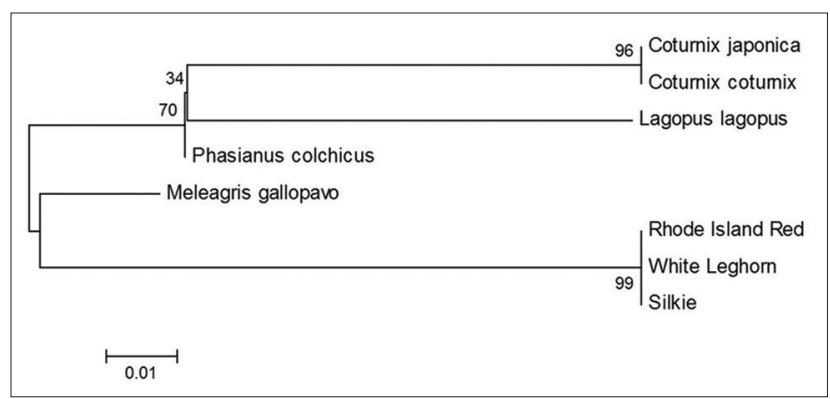

Figure-12: Phylogenetic tree based on 176 bp fragment nucleotide sequence of $M \times 1$ gene.

chicken in another genus Gallus of one family Phasianidae [14].

\section{Conclusion}

The $M x 1$ gene was found to be polymorphic in Japanese quail in one of the four fragments studied. The B allele was predominant, out of the three alleles found in $148 \mathrm{bp}$ fragment of $M x 1$ gene. Analysis of four different fragments showed that Japanese quail Mxl gene showed relatively high degree of homology with other poultry species. The relative conserve nature of $M x l$ gene across the species confirms its biological role as an immunity-related gene. The other regions of this gene need to be sequenced and association with disease resistance traits may be done for the complete characterization of this gene in Japanese quail.

\section{Authors' Contributions}

$\mathrm{PK}, \mathrm{BB}, \mathrm{TKB}$, and $\mathrm{AKT}$ planned and designed the experiment. DK conducted the experimental work. RN and VKS collected the blood samples. CM, NRS, $\mathrm{KS}$, and DS were involved in scientific discussion and analysis of the data. All authors read and approved the final manuscript.

\section{Acknowledgments}

The authors are thankful to the Director, Indian Veterinary Research Institute (IVRI), Izatnagar, Bareilly, India for providing necessary facilities to carry out this work.

\section{Competing Interests}

The authors declare that they have no competing interests.

\section{References}

1. Thornton, P.K. (2010) Livestock production: Recent trends, future prospects. Philos. Trans. Royal Soc. B. Biol. Sci., 365: 2853-2867.

2. Thompson-Crispi, K.A., Sargolzaei, M., Ventura, R., AboIsmail, M., Miglior, F., Schenkel, F. and Mallard. B.A. (2014) A genome-wide association study of immune response traits in Canadian Holstein cattle. BMC Genom., 15: 559 .

3. Nikbakht, G. and Esmailnejad, A. (2015) Chicken major histocompatibility complex polymorphism and its assoc ation with production traits. Immuno Genet., 67(4): 247

4. Jie, H. and Liu, Y.P. (2011) Breeding for disease resistance in poultry: Opportunities with challenges. World's Poultr. Sci. J., 67(4): 687.

5. Sambrook, J. and Russell, D.W. (2001) Molecular Cloning: A Laboratory Manual. ${ }^{\text {rd }}$ ed. Cold Spring Harbor Laboratory Press, New York, USA

6. Untergrasser, A., Cutcutache, I., Koressaar, T., Ye, J., Faircloth, B.C., Remm, M. and Rozen, S.G. (2012) Primer3 - New capabilities and interfaces. Nucl. Acid. Res., 40(15): e115.

7. Available from: https://eu.idtdna.com/Primerquest/Home/ Index. Accessed on 18-06-2015.

8. Bassam, B.J., Caetano-Anolles, G. and Gresshoff, P.M. (1991) Fast and sensitive silver staining of DNA in polyacrylamide gels. Anal. Biochem., 196: 80-83.

9. Orita, M., Iwahana, H., Kanazawa, H., Hayashi, K. and Sekiya, T. (1989) Detection of polymorphisms of human DNA by gel electrophoresis as single strand conformation polymorphisms. PNAS USA, 86: 2766-2770.

10. Mishra, C., Das, D., Kumar, P., Khanna, K., Singh, A.P., Dayal, S., Selvaramesh, A.S, Bhattacharya, T.K., Bhushan, B. and Sharma, A. (2011) Nucleotide sequencing and PCR-SSCP of $M x 1$ gene in chicken. Indian. J. Anim. Res., 45(4): 276-282.

11. Hall, T. (2011) BioEdit: An important software for molecular biology. GERF Bull. Bio., 2: 60-61.

12. Tamura, K., Stecher, G., Peterson, D., Filipski, A. and 
Kumar, S. (2013) MEGA6: Molecular evolutionary genetics analysis version 6.0. Mol. Bio. Evol., 30: 2725-2729.

13. Sanger, F., Nicklen, S. and Coulson, A.R. (1997) DNA sequencing with chain terminating inhibitors. PNAS USA,
74: 5463-5467.

14. Dickinson, E.C., Christidis, L and Jr. Remsen, J.V. (2003) The Howard and Moore Complete Checklist of the Birds of the World. Christopher Helm, London.

$* * * * * * * *$ 